\title{
Conocimientos prácticos sobre neuropatía diabética en médicos especialistas en medicina familiar y residentes de una Unidad de Medicina Familiar
}

\author{
Saúl Guzmán-Herrera, Guillermo Muñoz-Zurita, Elías Pezzat-Zaid \\ Facultad de Medicina de la Benemérita Universidad Autónoma de Puebla (FMBUAP)
}

\section{RESUMEN}

Introducción. La neuropatía diabética se define como el conjunto de alteraciones morfológicas y funcionales del sistema nervioso periférico causado por la diabetes, cuando no existen otras causas de neuropatía. Se estima que la prevalencia e incidencia de la neuropatía diabética clínicamente significativa es del 60\%. Objetivo. Determinar el manejo y nivel de conocimientos que sobre neuropatía diabética, tienen los médicos que laboran en el primer nivel de atención, en la Unidad de Medicina Familiar número 2 del Instituto Mexicano de Seguro Social de la ciudad de Puebla.

Materiales y Métodos. Se trata de un estudio descriptivo, observacional, homodémico, trasversal y prospectivo en 72 médicos, de los cuales 32 son médicos especialistas en medicina Familiar y 40, residentes de la Unidad de Medicina Familiar número 2, a los que se les aplicó una encuesta para la determinación de conocimientos sobre neuropatía diabética.

Resultados. De la población total encuestada, $59.7 \%$ consideraron que la afectación principal de la neuropatía diabética es en el SNP, solo $37.5 \%$ consideraron que el principal factor de riesgo de la neuropatía diabética es la hiperglucemia prolongada; $67 \%$ establece su diagnóstico en base a la exploración física y la anamnesis; solo
$28.1 \%$ tiene presente que el medicamento de primera línea son los antidepresivos tricíclicos; $16 \%$ conoce un test validado para el diagnóstico de neuropatía diabética.

Conclusiones. El conocimiento sobre la neuropatía diabética en los médicos de primer nivel de atención es limitado.

Palabras Clave: conocimientos, neuropatía diabética, medicina familiar.

\section{ABSTRACT \\ Practical Knowledge of Diabetic Neuropathy in Family Medicine Physicians and Residents of a Family Medicine Unit}

Introduction. Diabetic neuropathy is defined as the set of morphological and functional alterations of the peripheral nervous system caused by diabetes when no other causes of neuropathy are found. It is estimated that the prevalence and incidence of clinically significant diabetic neuropathy is $60 \%$.

Objective. To determine the management and knowledge of diabetic neuropathy by doctors working in primary care in the Family Medicine Unit No. 2 of the IMSS hospital in the city of Puebla.

Materials and Methods. This was a descriptive,

Autor para correspondencia: Dr Sául Guzmán-Herrera, Departamento de Agentes Biológicos, Facultad de Medicina, Benemérita Universidad Autónoma de Puebla. Calle 13 Sur No.2702, Puebla, Puebla, México. E-mail: saulguzman71@gmail.com

Recibido: el 18 de junio de 2014. Aceptado para publicación: el 24 de noviembre de 2014

Este documento está disponible en http://www.revbiomed.uady.mx/pdf/rb152612.pdf

Vol. 26, No. 1, enero-abril de 2015 
Guzmán-Herrera et al.

observational, homodemic, prospective cross investigation, with participation by 72 physicians, including 32 Family Medicine physicians and 40 Residents in the Family Medicine Unit No. 2. Participants were given a survey to assess their knowledge about diabetic neuropathy. descriptive Results. Of the total population surveyed, $59.7 \%$ felt that the main involvement of diabetic neuropathy is in the peripheral nervous system, while only $37.5 \%$ considered prolonged hyperglycemia as the main risk factor for diabetic neuropathy, and 67\% made their diagnoses based on exploration anamnesis and physical exams only, while only $28.1 \%$ were aware of tricyclic antidepressants as elective treatments. Only $16 \%$ knew of a validated test for the diagnosis of diabetic neuropathy.

Conclusion. Knowledge about diabetic neuropathy in the first level of medical care is limited.

Key words: knowledge, diabetic neuropathy, family medicine

\section{INTRODUCCIÓN}

La neuropatía diabética se define como el conjunto de alteraciones morfológicas y funcionales del sistema nervioso periférico, causada por la diabetes cuando no existen otras causas de neuropatía; incluye un grupo de síndromes clínicos con manifestaciones que involucra nervios periféricos, somáticos y autonómicos (1). Se estima que la prevalencia e incidencia de la neuropatía diabética clínicamente significativa es de $60 \%$; sin embargo, se puede demostrar alteraciones electrofisiológicas en casi 100\% de los diabéticos; aunque, en muchos de estos pacientes, la neuropatía es subclínica $(1,2)$.

La neuropatía dolorosa es la complicación con mayor impacto en el área física, psicológica y económica; la prevalencia es de $8 \%$ en pacientes diabéticos recién diagnosticados y más de $50 \%$ en pacientes con larga evolución (1-3).
La hiperglucemia, que afecta a los microvasos sanguíneos, sería el desencadenante primordial de la neuropatía diabética dolorosa (NPD) (4). Las alteraciones principales son la reduplicación de la membrana basal, la oclusión vascular, que altera la barrera entre las fibras nerviosas y los microvasos del endoneuro y del perineuro por lo que los síntomas, usualmente, están confinados a una parte o a la totalidad del territorio de inervación de la estructura nerviosa afectada (4-5). La exploración física revela alteraciones iniciales como dolor quemante, paresias y parestesias, así como, pérdida sensitiva con distribución en guante o calcetín; sin embargo, en etapas tardías puede llegar a afectar el componente motor de los nervios e, inclusive, el componente autonómico de otros sistemas (p.e. gastroparesia diabética).

Para realizar una exploración completa, se debe emplear una torunda de algodón seca, un palillo de dientes de madera o similar, un objeto frío y otro caliente y un diapasón de 128 Hz (6-9).

El manejo inicial incluye cambios en el estilo de vida que impacten en el control glucémico, como son, el cuidado de los pies y tratamiento farmacológico dentro del cual se incluyan antidepresivos tricíclicos y la pregabalina de primera línea, pudiéndose emplear también inhibidores selectivos de la recaptura de serotonina (ISRS), opioides e incluso anticonvulsionantes (17).

La literatura médica, en cuanto al conocimiento del médico acerca de neuropatía diabética es escasa y, debido a que en nuestra investigación no encontramos un estudio similar, el objetivo de nuestro estudio es evaluar los conocimientos acerca de la neuropatía diabética con que cuentan los médicos familiares de primer contacto, así como, los residentes de una unidad de medicina familiar en la ciudad de Puebla, México.

\section{MATERIALES Y MÉTODOS}

Se trata de un estudio descriptivo,

\section{Revista Biomédica}


Niveles de conocimientos de neuropatía diabética

observacional, homodémico, trasversal y prospectivo, realizado en la Unidad de Medicina Familiar número 2 de la ciudad de Puebla, donde laboran 92 médicos, de los cuales 51 son médicos especialistas en medicina familiar y 41 médicos residentes en formación. El personal encuestado incluyó médicos con especialidad en medicina familiar, o estuvieran cursando la residencia en la misma; que laboraran ya sea en el turno matutino o vespertino; y, que aceptaran voluntariamente, contestar la encuesta; se encuestó 32 médicos especialistas en medicina familiar $(62.7 \%$ del total de médicos que labora en dicha institución) y 40 médicos residentes de la misma especialidad (97.1\% del total de médicos residentes en formación); en este último grupo, 15 fueron de primer año $(100 \%$ de los residentes de primer año), 16 de segundo año (100\% de los residentes de segundo año) y 9 de tercer año. (90\% de los médicos residentes del tercer año). Se les aplicó una encuesta, previamente verificada por 3 especialistas en el área, la cual incluyó un breve apartado en el que se indagaron, tanto los años de egreso de la facultad de medicina de los médicos de base, como su antigüedad laboral en la unidad de medicina familiar No. 2.

El apartado siguiente se diseñó para evaluar el conocimiento acerca de la neuropatía diabética periférica, para lo cual se incluyeron 10 reactivos que abarcaran definición, epidemiologia, patogenia, diagnóstico y tratamiento acerca de dicha enfermedad. La encuesta fue anónima, y se solicito su consentimiento para el análisis y la divulgación de los resultados obtenidos por el protocolo.

\section{RESULTADOS}

El instrumento de evaluación se aplicó a 72 médicos; 32 médicos especialistas en medicina familiar y 40 residentes de la misma especialidad. El promedio de años de egreso de la escuela de medicina en médicos de base fue de $87.5 \%$ para el rango de más de 10 años y, solo de $12.5 \%$ para el rango de 6-10 años. En cuanto a la antigüedad laboral en la Unidad de Medicina Familiar 2 (UMF 2), el grupo más numeroso (56.25\%) fue el de más de 10 años de servicio, mientras que, el porcentaje restante no rebasaba los 10 años de servicio. Todos los médicos seleccionados accedieron a participar en el estudio, por lo que no hubo reemplazos.

De la población total encuestada, solo $59.7 \%$ consideraron que el sistema nervioso periférico es el afectado; en la respuesta por grupos (el grupo de residentes y el grupo de médicos de base) $60 \%$ de los residentes y $59.37 \%$ de los médicos especialistas tuvieron la misma consideración. (Figura 1).

Al cuestionar acerca de las formas clínicas de neuropatía, 92\% del total de médicos encuestados dijo conocer la polineuropatías y mononeuropatía; 4\% mencionó las formas proximal y distal; 3\% respondió simétrica y asimétrica, mientras que, $1 \%$ no respondió el cuestionamiento. (Figura 2).

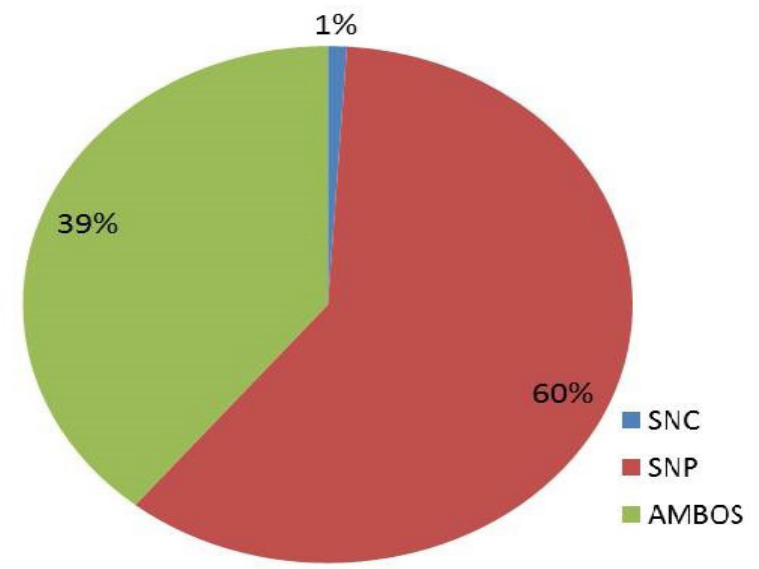

Figura 1. Área de afectación de la neuropatía diabética en el Sistema Nervioso

De los médicos encuestados, solo $37.5 \%$ consideró que el principal factor de riesgo para el desarrollo de la neuropatía diabética eran los niveles de hiperglucemia prolongada; en los resultados por grupos, $50 \%$ de los residentes y $21.87 \%$ de los médicos especialistas, tuvieron la 
Guzmán-Herrera et al.

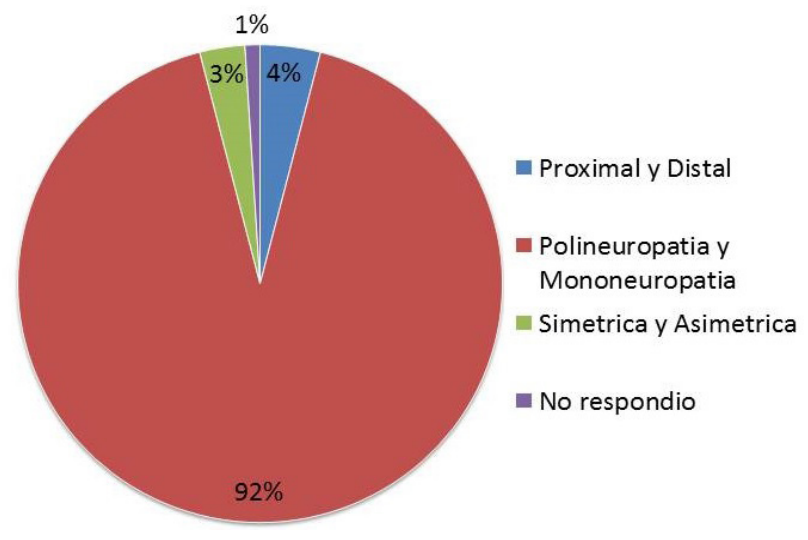

Figura 2. Formas clínicas de neuropatía más conocidas

misma conclusión.

En el cuestionamiento ¿Cuál es la vía hiperactivada en la patogenia de la neuropatía diabética?, 55\% del total de médicos consideraron que es la vía de los polioles, mientras que, 23\% no respondió. En el resultado por grupos, $42.5 \%$ del total de médicos residentes y $12.5 \%$ de los médicos de base, coincidieron con misma respuesta (Figura 3).

Del total de médicos encuestados, 69\% establece el diagnóstico de neuropatía diabética usando solamente monofilamento o similar; sin embargo, alrededor de $17 \%$ considera que no es necesario ningún instrumento para establecer el diagnóstico de neuropatía diabética periférica. En cuanto a los resultados por grupos, esta percepción es proporcionalmente similar entre el grupo de médicos especialistas y residentes (68.75\% y $62.5 \%$ respectivamente), quienes

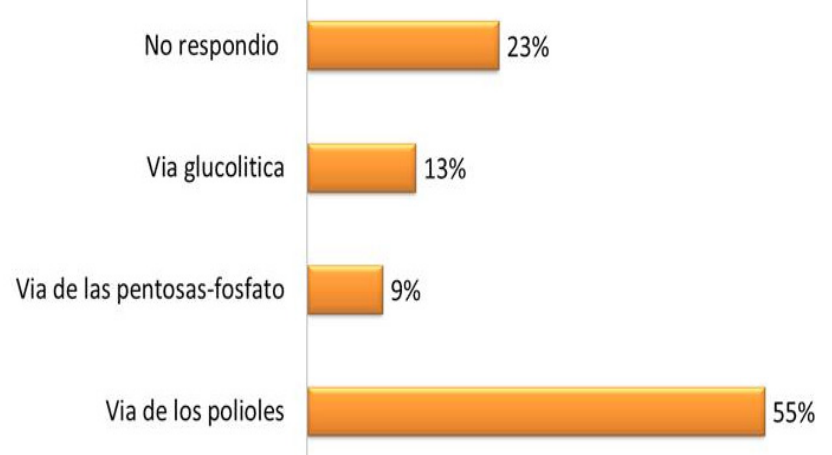

Figura 3. Porcentaje de repetición establecen el diagnóstico de neuropatía diabética usando solamente un monofilamento o similar. (Figura 4).

Al cuestionarles por la presentación clínica más frecuente, $47 \%$ indicó que la presentación más común en la consulta, es la de parestesias en guante o calcetín; 23\% refiere dar consulta a pacientes con manifestaciones de dolor de tipo ardoroso y $26 \%$ de los médicos mencionó que las parestesias y dolor tipo quemante fue el motivo más frecuente de consulta (Figura 5). Por otro lado, al cuestionarles acerca del método

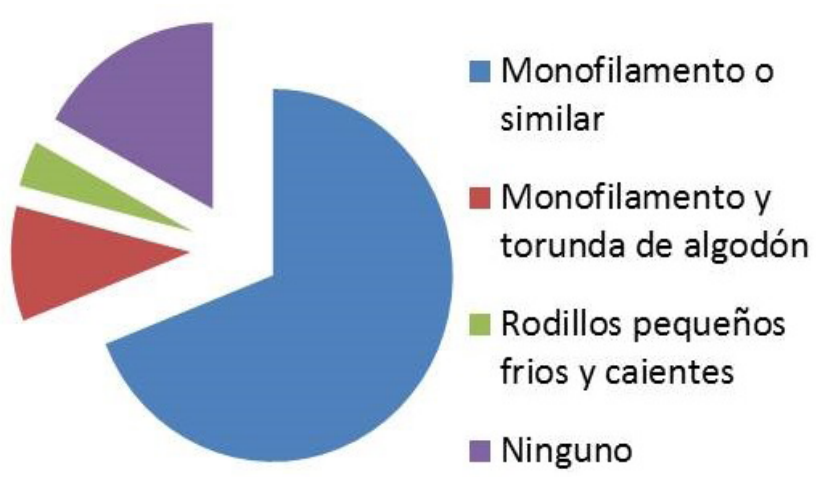

Figura 4. Instrumentos de diagnóstico de neuropatía diabética

diagnóstico confirmatorio de neuropatía diabética, 94\% mencionó la electromiografía como el principal estudio diagnostico confirmatorio de la alteración.

$32 \%$ de la población encuestada considera a los fármacos anticonvulsivantes como el tratamiento de primera línea, seguido por $31 \%$ de los encuestados quienes consideran en este rubro a los ISRS. Solo $20 \%$ de los encuestados consideró a los antidepresivos tricíclicos como el tratamiento de elección. Al comparar las respuestas entre residentes y médicos especialistas en medicina familiar, se observó que, en estos últimos, existe una mayor proporción respecto a sus compañeros especialistas en cuanto a considerar como medicamento de primera línea a los antidepresivos tricíclicos (28.1\%), a

\section{Revista Biomédica}


Niveles de conocimientos de neuropatía diabética

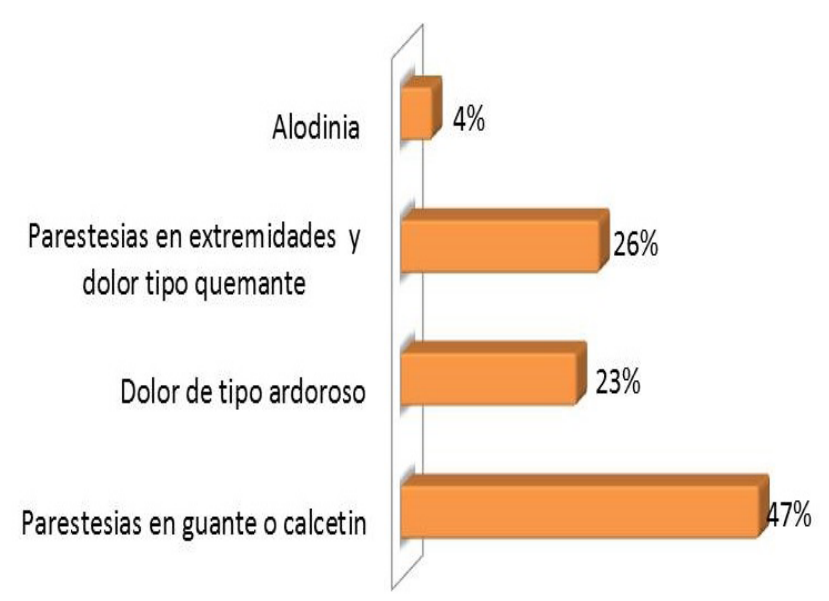

Figura 5. Manifestaciones clínicas más frecuentes en la neuropatía diabética

diferencia de los médicos residentes quienes, en su respuesta más repetida ( $45 \%$ ), consideran que son los anticonvulsivantes los medicamentos de primera línea (Figura 6).

Al hacer mención acerca de algún cuestionario validado y estandarizado para ser empleado en el diagnóstico y seguimiento de la neuropatía diabética periférica, $90.3 \%$ de la población total menciona no conocer ningúno, mientras que, $9.7 \%$ refirieró conocer el instrumento denominado Test de Michigan. Dentro este último porcentaje, 3 sujetos corresponden al grupo de los médicos especialistas y 6 corresponden al grupo de los residentes.

\section{DISCUSIÓN}

La literatura relacionada con la evaluación del conocimiento de neuropatía diabética dolorosa

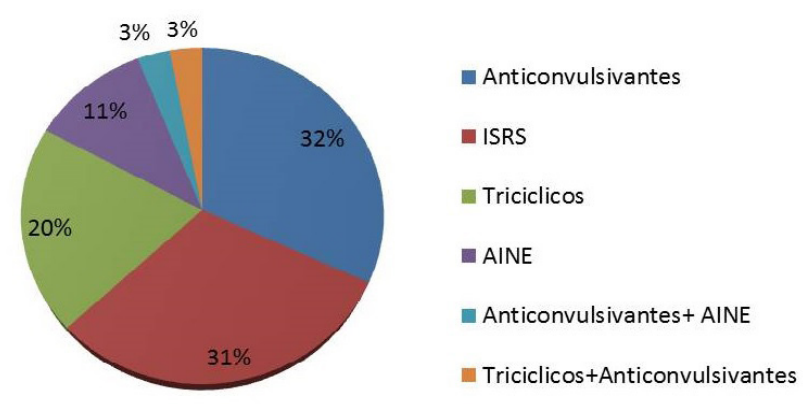

Figura 6. Fármacos utilizados con mayor frecuencia para el tratamiento de la neuropatía diabética es abundante en lo que se refiere al paciente; sin embargo, en cuanto al personal médico es escasa. Hasta donde se sabe, este es el primer informe en el que se describe la evaluación de conocimiento de neuropatía diabética dolorosa en médicos de primer nivel de atención a la salud en el que observamos, en general, falta de conocimiento para definir correctamente a la neuropatía diabética, y sus formas clínicas de presentación, tanto en médicos especialistas, como en médicos residentes. Por otro lado, la poca identificación del principal factor de riesgo (hiperglicemias prolongadas), nos permite inferir la baja sospecha que los médicos tienen para hacer el diagnóstico temprano de la NPD. El estudio nos permite deducir el bajo nivel de comprensión que la población encuestada tiene de la patogenia de esta complicación de la diabetes, lo cual es demostrado por el desconocimiento general que se tiene de la vía cuya afectación hace posible el desarrollo de la NPD.

El hecho de que $60 \%$ haya respondido que solo emplea el monofilamento en la evaluación del paciente con NPD, refleja la falta de conocimiento que se tiene de la correcta evaluación clínica del paciente, puesto que, el solo uso del monofilamento no tiene valores adecuados de sensibilidad y especificidad para usarlo como único auxiliar diagnóstico; sin embargo, también puede ser resultado de las carencias institucionales de otros insumos, como diapasón de $128 \mathrm{~Hz}$ o rodillos fríos y calientes.

La respuesta dada por los médicos encuestados, basándose en su experiencia, concuerda con la forma de presentación de neuropatía diabética más frecuente reportada por la literatura internacional, que es la presencia de parestesias en guante o calcetín, lo que bien se esperaba puesto que los estudios epidemiológicos así lo confirman. Al momento de abordar lo referente al tratamiento de primera línea, nos damos cuenta que es, quizá, una de las respuestas más dispares dentro de nuestra encuesta a pesar de que hace tiempo está bien estipulado que el 
Guzmán-Herrera et al.

tratamiento de primera línea para la neuropatía diabética deben ser los antidepresivos tricíclicos o la pregabalina. Al respecto, encontramos que, entre los médicos encuestados, un grupo numeroso considera a los anticonvulsivantes, a pesar de que estos son considerados por las guías internacionales como la tercera línea de tratamiento, lo que habla de la falta de información correcta en una proporción importante de médicos de esta clínica. Sin embargo, hay que señalar que esta falta de información está más acentuada en los médicos residentes, quienes en un porcentaje mayor, respondieron la opción de anticonvulsivantes; mientras que, la mayoría de los médicos especialistas señalaron a los antidepresivos tricíclicos

Se observó también que los instrumentos validados para la detección de la neuropatía diabética son desconocidos para gran parte de los médicos lo que puede enlentecer el correcto diagnóstico y el tratamiento adecuado que son parte esencial en el control de la neuropatía diabética. Es importante hacer mención que, entre los residentes, está más extendida la idea del uso de tests correctamente validados y estandarizados debido a su formación y su adecuación en las guías de práctica clínica y medicina basada en evidencias.

Finalmente, dentro de lo que se esperaba, se observó que 94\% reconoce que el estudio electromiográfico es el indicado para la confirmación del diagnóstico de NPD; sin embargo, esto resulta un tanto controversial puesto que en la práctica institucional es poco accesible y su uso está reservado para las formas atípicas de neuropatía.

\section{CONCLUSIONES}

El conocimiento que se tiene en relación a la neuropatía diabética, es deficiente en el grupo encuestado. Además, los resultados varían poco entre el grupo de médicos residentes y médicos especialistas por lo que se infiere que el desconocimiento es generalizado en la población encuestada y no es solo sectorial (médicos especialistas y residentes). Aunque, cabe señalar que el estudio se realizó en una clínica prototipo del Instituto Mexicano del seguro social, los resultados no pueden ser extrapolados al total de la población medica de la ciudad de puebla; sin embargo, el estudio puede ser ampliado para tener mejor validez e implementar en lo subsecuente un programa de capacitación dirigido a la población médica.

\section{AGRADECIMIENTOS}

Agradecemos al Dr. Rodríguez Aguilar José Emilio Gerardo, jefe de enseñanza de la UMF $N^{\circ} 2$, por las facilidades brindadas para la realización de este trabajo.

\section{REFERENCIAS}

1. Fernández Travieso Julio C. Manejo de la neuropatía diabética. Revista CENIC Ciencias Biológicas, Vol. 38, No. 1, 2007. Enero-Abril, 20-26.

2. Betancourt JA, Covarrubias GA, Guevara LU. Mecanismos generadores y consideraciones terapéuticas futuras de la polineuropatía diabética dolorosa. Rev Mex Anest 2008;31:28-36.

3. American Diabetes Association. (2014). Standards of medical care in diabetes-2014. Diabetes care, 37, S14.

4. Boulton AJM. Diabetic neuropathy: classification, measurement and treatment. Current Opinion in Endocrinology \& Diabetes; 2007. 141-145.

5. Pedraza C. Neuropatías diabéticas formas clínicas y diagnóstico. Rev. Med. Clin. CONDES; 2009. 20 (5) 681-686.

6. ALAD \& WORLD WIDE. Guía práctica en el Manejo de la Polineuropatía diabética. NEURALAND. 2010.

7. ACCORD. Effects of Intensive Glucose Lowering in Type 2 Diabetes. N Engl J Med.2008 358:2545-2559.

8. Brian C Callaghan, Hsinlin $T$ Cheng, Catherine $L$ Stables, Andrea L Smith, Eva L Feldman. Diabetic neuropathy: clinical manifestations and current treatments. The Lancet Neurology. June 2012. 521534.

9. Curià Casanoves Francisco J. Evidencias en el tratamiento del dolor neuropático. FMC. 2008. 15(9):592-604.

10. Samper Bernal, Monerris Tabasco MM, Homs Riera M. Etiología y manejo de la neuropatía diabética dolorosa. Rev. Soc. Esp. Dolor. 2010. 17(6):286-296.

\section{Revista Biomédica}


Niveles de conocimientos de neuropatía diabética

11. Haanpää Maija, Attal Nadine, Backonja Miroslav, et al. NeuPSIG guidelines on neuropathic pain assessment. PAIN. 152 14-27. 2011

12. Cruccu, Giorgio, and Andrea Truini. "Tools for Assessing Neuropathic Pain.” PLoS Medicine 6.4 (2009): e1000045. [En Linea] [Consultado 1 de Dic. 2013].

13. Fernández Y, Acuna A, Carrabs M. Análisis farmacoeconómico de Carbamazepina, Gabapentina, Pregabalina en el manejo del dolor neuropático. Informe Médico 2009. 11 (6): 335-340.

14. Attal, N, Cruccu, G, Baron, R, Haanpää, M., Hansson, P, Jensen, T. S, et al (2010). EFNS guidelines on the pharmacological treatment of neuropathic pain: 2010 revision. European Journal of Neurology, 17(9), 1113-e88.

15. Secretaría de Salud, Guía de Práctica Clínica: Diagnóstico, y tratamiento médico del dolor por neuropatía diabética en adultos en el primer nivel de atención, México: , 2009.

16. Lindsay TJ1, Rodgers BC, Savath V, Hettinger K. Treating diabetic peripheral neuropathic pain. Am Fam Physician. 2010 Jul 15;82(2):151-8.

17. Feldman EL, McCulloch DK. Treatment of diabetic neuropathy. Last reviewed November 2014. UpToDate Inc. Waltham, MAPsychopharmacol Bull 2003;37(4):99-115. 